Case Report

\title{
Histiocytic Sarcoma Associated with Coombs Negative Acute Hemolytic Anemia: A Rare Presentation
}

\author{
Sandeep Batra, ${ }^{1}$ Stephen C. Martin, ${ }^{1}$ Mehdi Nassiri, ${ }^{2}$ Amna Qureshi, ${ }^{2}$ and Troy A. Markel ${ }^{3}$ \\ ${ }^{1}$ Department of Pediatrics, Section of Pediatric Hematology and Oncology, Riley Hospital for Children at Indiana University Health, \\ Indiana University School of Medicine, Indianapolis, IN 46202, USA \\ ${ }^{2}$ Department of Pathology and Laboratory Medicine, Indiana University School of Medicine, Indianapolis, IN 46202, USA \\ ${ }^{3}$ Department of Surgery, Section of Pediatric Surgery, Riley Hospital for Children at Indiana University Health, \\ Indiana University School of Medicine, Indianapolis, IN 46202, USA \\ Correspondence should be addressed to Sandeep Batra; batras@iu.edu
}

Received 11 February 2016; Revised 22 May 2016; Accepted 1 June 2016

Academic Editor: Jose I. Mayordomo

Copyright (C) 2016 Sandeep Batra et al. This is an open access article distributed under the Creative Commons Attribution License, which permits unrestricted use, distribution, and reproduction in any medium, provided the original work is properly cited.

Histiocytic sarcoma (HS) rarely involves extranodal sites, such as the spleen. We report a unique pediatric case of massive splenomegaly and refractory Coombs negative hemolytic anemia (CNHA) secondary to HS. The CNHA resolved completely after an emergent splenectomy. Next generation sequencing (NGS) revealed novel ASXL1, PTPN11, KIT, and TP53 mutations, unmasking a clonal heterogeneity within the same neoplasm.

\section{Introduction}

Malignant histiocytic disorders such as histiocytic sarcoma (HS) are rare in the pediatric population [1]. HS commonly presents with fever, malaise, weight loss, and abdominal pain, but clinical manifestations are varied. HS can be localized or fulminant and uncommonly involves extranodal sites such as skin, bone marrow, soft tissue, and spleen [2]. Fulminant or disseminated HS is often associated with a poor outcome [2].

HS may occur before or after mature B cell lymphomas, acute lymphoblastic, and hairy cell leukemia, suggesting that a common oncogenic cellular origin may exist in some patients $[3,4]$. HS can also be associated with autoimmune lymphoproliferative syndrome [5]. The majority of HS express macrophage or histiocytic markers such as lysozyme, alpha-antitrypsin, CD68 (KP1 and PGM1), CD163, CD11c, and CD14 but typically lack Langerhans cell (CDla and langerin), follicular dendritic cell (CD21 and CD35), and myeloid cell (CD33, CD13, and myeloperoxidase) markers. CD34, CD3, and CD20 (T and B lymphocyte markers, resp.), melanoma (Melan-A, human melanoma black-45, and tyrosinase), and carcinoma (cytokeratin) markers are typically absent [6]. Rarely lineage infidelity may be present and could lead to misdiagnosis of a lymphoma [7].
We report a case of a 17-year-old who presented with massive splenomegaly and severe Coombs negative hemolytic anemia (CNHA). The diagnosis of histiocytic sarcoma (HS) was established after an emergent splenectomy. To our knowledge, HS associated with CNHA in the pediatric population has not been previously reported.

\section{Case Report}

A 17-year-old African American male presented to the local emergency department with fatigue, abdominal pain, jaundice, and worsening pallor, for 2 weeks. On exam, he was pale and icteric and had tender splenomegaly (spleen was palpable $5-6 \mathrm{~cm}$ below costal margin). Labs revealed a hematocrit equal to $17 \%$, serum bilirubin equal to $3 \mathrm{mg} / \mathrm{dL}$, a negative Coombs test (direct and indirect), and a platelet count of 39,000 . The peripheral smear showed anisopoikilocytosis, abundant spherocytes, tear drop cells, polychromatophilia, with normal appearing granulocytes, and decreased platelets (Figure 2(a)). A bone marrow aspirate revealed a hypercellular marrow, negative for blasts, dysplasia, or hemophagocytosis. The local hematologist initiated treatment with oral prednisone $(2 \mathrm{mg} / \mathrm{kg} /$ day $)$ and weekly rituximab $\left(375 \mathrm{mg} / \mathrm{m}^{2}\right.$ for 4 total doses) and administered 2 doses of intravenous 


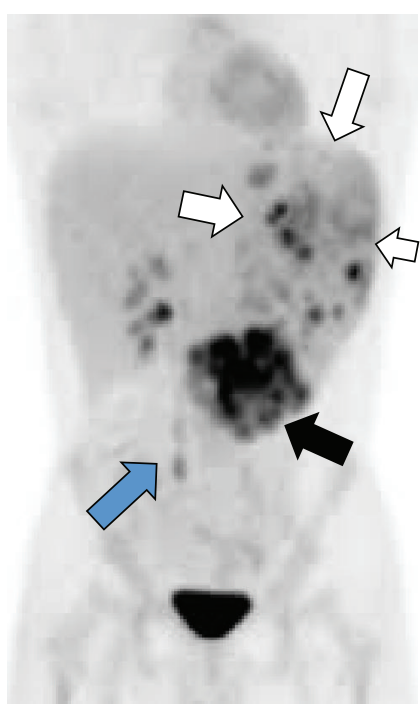

(a)

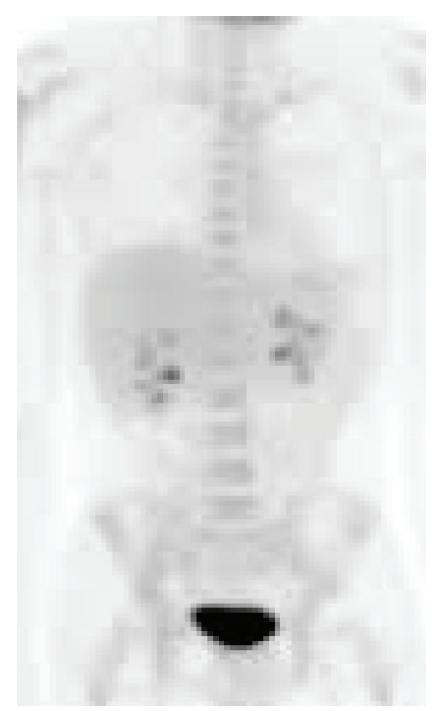

(b)

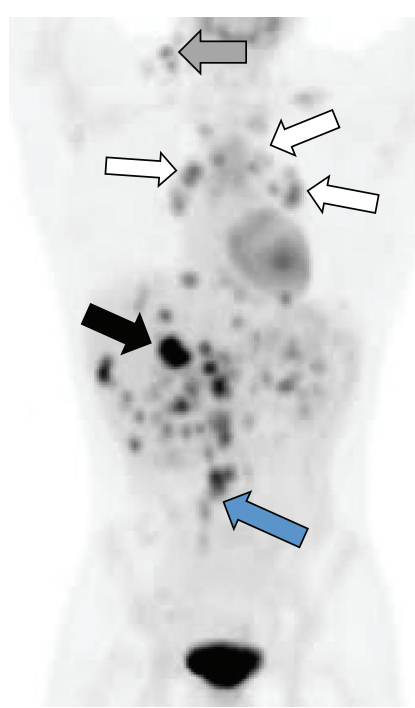

(c)

FIGURE 1: PET scans. (a) Prechemotherapy PET-CT scan (anterior view) demonstrates marked splenomegaly (white arrows), with multiple hypermetabolic foci scattered throughout the spleen and in the retroperitoneum (blue arrow). There was a large aggregate of abnormal foci at the lower edge of the spleen that measured $7 \times 6 \times 5 \mathrm{~cm}$ (black arrow); (b) PET-CT after 6 cycles of CHOP chemotherapy shows no evidence of disease; (c) recurrent hypermetabolic foci and lymphadenopathy involving multiple sites within the liver (black arrow), peritoneum (blue arrow), mediastinum (white arrows), and neck (gray arrow).

immunoglobulin (1 gram/kilogram body weight given a week apart), based on the presumptive diagnosis of a Coombs negative Evans Syndrome.

However, the hemolytic anemia persisted, and the patient continued to require 2-4 units of packed RBC transfusions to keep the hematocrit $>25 \%$. The spleen (now below umbilicus) continued to increase in size and was associated with severe abdominal pain and distension. He was then referred to our hematology service for further evaluation. We obtained additional labs as follows: ALPS panel (negative), cold agglutinins (negative), reticulocyte count (10\%), indirect and direct Coombs testing (negative), bilirubin ( $5 \mathrm{mg} / \mathrm{dL}$, mostly indirect), and serum haptoglobin (undetectable). Flow cytometry on peripheral blood failed to reveal an abnormal blast population and demonstrated a normal RBC expression pattern for CD55 or CD59, excluding acute leukemia or $\mathrm{PNH}$, respectively, as the probable cause of the presentation. The urinalysis was negative for hemosiderin or blood. Infectious work-up for Bartonella, brucellosis, tuberculosis, and malaria was negative. We obtained an abdominal PET-CT which demonstrated marked splenomegaly, with multiple areas of heterogeneous and hypermetabolic enhancing foci/masses that were scattered throughout the spleen and also in the retroperitoneum (Figure 1(a)). These findings were suggestive of a lymphoproliferative and neoplastic process.

An urgent open splenectomy was performed. The surgically removed spleen (Figure 2(b)) was massively enlarged (weight $=1770$ grams, $24 \times 14 \times 11 \mathrm{~cm}$ in size) and very firm in consistency. There were several hypertrophied vessels noted in the splenic hilum. Serial sections revealed a redbrown, congested parenchyma, with numerous gray-yellow nodules $(0.1-2 \mathrm{~cm}$ in size), concentrated at the lower edge of the spleen. A repeat bone marrow aspirate demonstrated a hypercellular marrow, with trilineage maturation and devoid of HS or hemophagocytosis.

Histological sections of the spleen revealed sheets of atypical multinucleated cells with a high nuclear to cytoplasmic ratio, irregular to round nuclear contour, prominent nucleoli, and an abundant eosinophilic cytoplasm (Figure 2(c)), with varying degrees of apoptosis. These neoplastic cells stained positively for CD45, CD68 PGM and Kp1 (Figures 2(d) and 2(e), resp.), CD14, CD23, fascin, and lysozyme (Figure 2(f)) and were negative for CD34, myeloperoxidase, and S100 protein, confirming the diagnosis of HS [7]. CD20, Pax5, CD3, CD30, keratin cocktail, desmin, factor VIII, factor XIIIa, CD163, CD1a, CD21, CD35, CD123 immunostains, and $\mathrm{BRAF}^{\mathrm{V} 600 \mathrm{E}}$ mutation were negative.

We also performed hotspot mutation detection by highthroughput next generation sequencing (NGS), using optimized oligonucleotide probes [8]. Specimens were reviewed by a pathologist before processing. Formalin-fixed paraffinembedded splenic tissue obtained at the time of diagnosis was used to isolate DNA. Fifteen full genes (exons only) as well as additional 39 oncogenic hotspots were analyzed with highly multiplexed next generation sequencing (Illumina TruSight Myeloid Sequencing Panel) (Supplemental Table 1 in Supplementary Material available online at http://dx.doi.org/10.1155/2016/3179147) [8]. Limit of detection of this assay as established by our laboratory is $1-2 \%$ mutant alleles. The variants were classified according to previously published guidelines and databases. Low prevalence $(<10 \%$ of allele frequency) mutations were detected in the ASLX1, KIT, PTPN11, and TP53 genes (Supplemental Table 2), along with multiple variants of unknown significance (not included in 


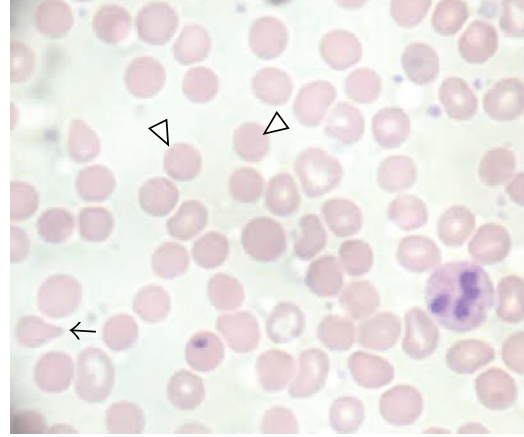

(a)

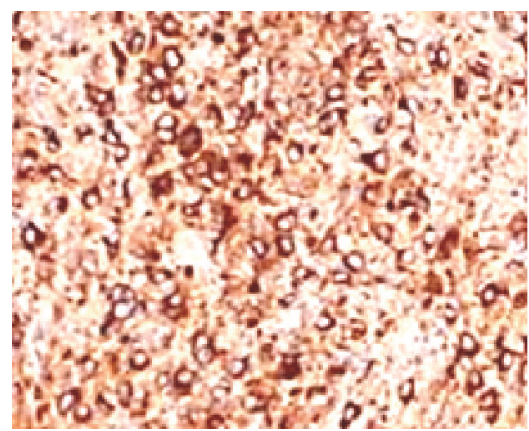

(d)

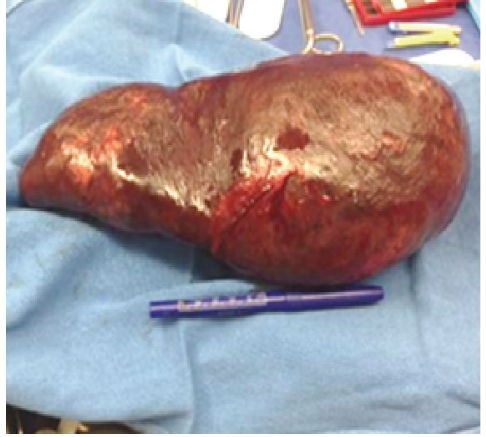

(b)

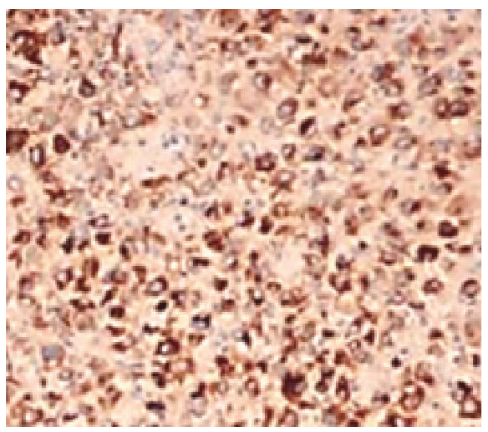

(e)

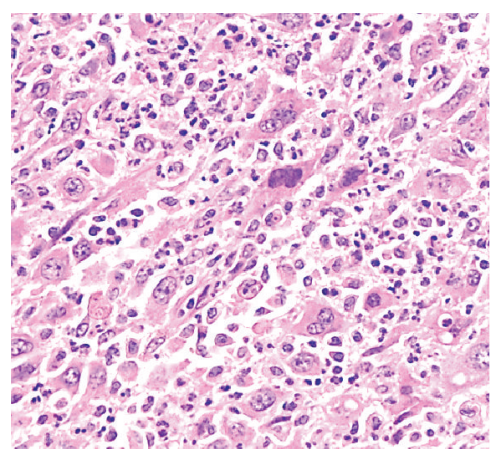

(c)

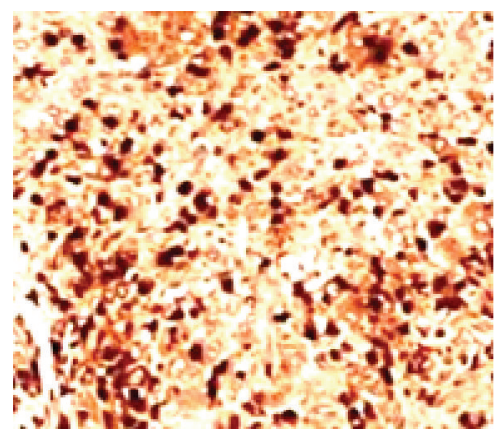

(f)

FIGURE 2: Peripheral blood smear and histopathology of the spleen. (a) Prechemotherapy peripheral blood smear with anisopoikilocytosis, numerous spherocytes (white arrows), tear drop cell (black arrow), and decreased platelets; (b) massively enlarged, surgically removed spleen (weight $=1770$ grams; $24 \times 14 \times 11 \mathrm{~cm}$ in size); (c) histological sections of the spleen revealed sheets of atypical cells with a high nuclear to cytoplasmic ratio, prominent nucleoli, and an abundant eosinophilic cytoplasm (400x magnification); (d, e, and f) areas of spleen involved with HS demonstrated strong and diffuse immunoreactivity with macrophage-specific markers (CD68 PGM (d), CD68 KP1 (e), and lysozyme (f)) [7].

supplemental data), suggesting that mutated or variant tumor cells comprised only a minor portion of the malignant clone.

Importantly, the hemolytic anemia and thrombocytopenia resolved rapidly and completely after splenectomy. We then elected to treat our patient with adjuvant CHOP chemotherapy (cyclophosphamide, doxorubicin, vincristine, and oral prednisone) $[9,10]$. The PET-CT scans after 3 cycles of CHOP and at the end of therapy (Figure 1(b)) demonstrated no residual or recurrent FDG avid lesions. However, the remission only lasted 3-4 months; recurrent disease was identified in the liver and abdominal lymph nodes (Figure 1(c)) on a PET scan and confirmed with a supraclavicular (neck) lymph node and CT guided liver needle biopsy. Interestingly, the CNHA did not recur with the relapse.

The relapsed disease was treated with 4 cycles of ICE (ifosfamide, carboplatin, and etoposide) chemotherapy [11] followed by an autologous stem cell transplant (using BEAM, carmustine, etoposide, cytarabine, and melphalan, as a conditioning regimen) for consolidation [12]. Unfortunately, our patient remained in remission only for 5-6 months, after transplant, and elected for palliative care.

\section{Discussion}

Acquired Coombs negative hemolytic anemia (CNHA) is rare in the adolescent age group. The differential diagnosis of CNHA (nonimmune hemolysis) associated with splenomegaly, in pediatric patients, includes both inherited and acquired causes such as hemoglobinopathies, RBC enzyme or membrane defects, infections, toxins, HLH, immunodeficiencies, microangiopathies, and uncommonly neoplasms $[13,14]$. Splenectomy is rarely performed in these patients but may be indicated in severe refractory immune mediated hemolysis, recurrent splenic sequestration, or hypersplenism [15].

Our patient presented with a Coombs negative hemolytic anemia (CNHA), associated with massive splenic involvement by HS. To our knowledge, this is the first pediatric report describing this atypical presentation. The CNHA could have resulted from the altered circulation, hypoxia, and acidification in the massive spleen. An emergent total splenectomy led to a rapid resolution of CNHA, supporting that notion.

Treatment with rituximab, IVIG, and steroids did not improve the CNHA. This initial lack of response to rituximab is, most likely, due to lack of CD20 expression or the existence of a nonimmune mechanism, such as the extravascular destruction of RBCs in the spleen, or direct cell mediated cytotoxicity $[16,17]$. There was no lab evidence of significant microangiopathy (no RBC fragments or schistocytosis) or hemophagocytic lymphohistiocytosis (HLH) (hemophagocytosis, hypertriglyceridemia, low NK activity, or hypofibrinogenemia) [18]. In addition, NGS failed to identify high 
prevalence $(>10 \%)$ oncogenic mutations that could explain the pathogenesis or the unique presentation of this HS.

Interestingly, the HS infiltrated spleen harbored mutations in the ASLX1, KIT, PTPN11, and TP53 genes (Supplemental Table 2). Mutations in ASXL1 have been observed frequently in acute myelogenous leukemia and myelodysplastic syndromes and are associated with a worse outcome due to an aberrant hematopoiesis [19]. Genomic profiling of acute myelogenous leukemia has identified somatic variants in both PTPN11 and KIT genes [20] and TP53 mutations [21], implicating resistant pathways that require further investigation [22]. It is plausible that these cooperating mutations contributed to HS relapse, in our patient.

\section{Conclusion}

This case report underscores the importance of ruling out an occult malignancy as a rare cause of refractory CNHA. Splenectomy is an effective option to treat CNHA due to splenic involvement by HS.

\section{Abbreviations}

HS: Histiocytic sarcoma

CNHA: Coombs negative hemolytic anemia

PET-CT: Positron emission tomography-computed tomography

ALPS: Autoimmune lymphoproliferative syndrome

CHOP: Cyclophosphamide, doxorubicin, vincristine, and prednisone

ICE: Ifosfamide, carboplatin, and etoposide

BEAM: Carmustine, etoposide, cytarabine, and melphalan

CD: $\quad$ Cluster of differentiation

BRAF: Serine/threonine-protein kinase B-Raf

HLH: Hemophagocytic lymphohistiocytosis

NGS: Next generation sequencing.

\section{Competing Interests}

The authors declare no conflict of interests.

\section{References}

[1] J. L. Heath, S. E. Burgett, A. M. Gaca, R. Jaffe, and D. S. Wechsler, "Successful treatment of pediatric histiocytic sarcoma using abbreviated high-risk leukemia chemotherapy," Pediatric Blood and Cancer, vol. 61, no. 10, pp. 1874-1876, 2014.

[2] J. L. Hornick, E. S. Jaffe, and C. D. M. Fletcher, "Extranodal histiocytic sarcoma: clinicopathologic analysis of 14 cases of a rare epithelioid malignancy," American Journal of Surgical Pathology, vol. 28, no. 9, pp. 1133-1144, 2004.

[3] P. Brunner, A. Rufle, S. Dirnhofer et al., "Follicular lymphoma transformation into histiocytic sarcoma: indications for a common neoplastic progenitor," Leukemia, vol. 28, no. 9, pp. 19371940, 2014.

[4] E. C. C. Castro, C. Blazquez, J. Boyd et al., "Clinicopathologic features of histiocytic lesions following ALL, with a review of the literature," Pediatric and Developmental Pathology, vol. 13, no. 3, pp. 225-237, 2010.

[5] C. Guitton, F. Le Deist, and B. Bader-Meunier, “Coombs' negative haemolytic anaemia as a first manifestation of autoimmune lymphoproliferative disease," British Journal of Haematology, vol. 129, no. 3, pp. 442-443, 2005.

[6] E. Takahashi and S. Nakamura, "Histiocytic sarcoma: an updated literature review based on the 2008 WHO classification," Journal of Clinical and Experimental Hematopathology, vol. 53, no. 1, pp. 1-8, 2013.

[7] S. A. Pileri, T. M. Grogan, N. L. Harris et al., "Tumours of histiocytes and accessory dendritic cells: an immunohistochemical approach to classification from the international lymphoma study group based on 61 cases," Histopathology, vol. 41, no. 1, pp. 1-29, 2002.

[8] C. H. Au, A. Wa, D. N. Ho, T. L. Chan, and E. S. K. Ma, "Clinical evaluation of panel testing by next-generation sequencing (NGS) for gene mutations in myeloid neoplasms," Diagnostic Pathology, vol. 11, no. 1, article 11, 2016.

[9] H. Tsujimura, T. Miyaki, S. Yamada et al., "Successful treatment of histiocytic sarcoma with induction chemotherapy consisting of dose-escalated CHOP plus etoposide and upfront consolidation auto-transplantation," International Journal of Hematology, vol. 100, no. 5, pp. 507-510, 2014.

[10] Y. Kitano, M. Nakagawa, M. Kojima et al., "Case of malignant histiocytosis treated with chop therapy and splenectomy," Nihon Naika Gakkai Zasshi, vol. 83, no. 6, pp. 990-992, 1994.

[11] J. Tomlin, R. K. Orosco, S. Boles et al., "Successful treatment of multifocal histiocytic sarcoma occurring after renal transplantation with cladribine, high-dose cytarabine, G-CSF, and mitoxantrone (CLAG-M) followed by allogeneic hematopoietic stem cell transplantation," Case Reports in Hematology, vol. 2015, Article ID 728260, 6 pages, 2015.

[12] B. Cazin, N. C. Gorin, J. P. Jouet et al., "Successful autologous bone marrow transplantation in second remission of malignant histiocytosis," Bone Marrow Transplantation, vol. 5, no. 6, pp. 431-433, 1990.

[13] V. Cecinati, F. Brugnoletti, M. D’Angiò et al., "Autoimmune hemolytic anemia and immune thrombocytopenia as unusual presentations of childhood hodgkin lymphoma: a case report and review of the literature," Journal of Pediatric Hematology/Oncology, vol. 34, no. 4, pp. 280-282, 2012.

[14] L. Uzunova, C. E. Hook, M. Gattens, and G. A. Burke, "Cold antibody autoimmune haemolytic anaemia in a child with diffuse large B cell lymphoma," Annals of Hematology, vol. 95, no. 1, pp. 151-152, 2016.

[15] S. Patel, J. Said, S. Song, W. Nishimoto, and R. Paquette, "A case of hemolytic anemia and severe thrombocytopenia related to histiocytic sarcoma," Leukemia Research, vol. 34, no. 9, pp. e257e258, 2010.

[16] E. Biagi, G. Assali, F. Rossi, M. Jankovic, B. Nicolini, and A. Balduzzi, "A persistent severe autoimmune hemolytic anemia despite apparent direct antiglobulin test negativization," Haematologica, vol. 84, no. 11, pp. 1043-1045, 1999.

[17] A. Fujimi, Y. Kamihara, Y. Kanisawa et al., "Anti-erythropoietin receptor antibody-associated pure red cell aplasia accompanied by Coombs-negative autoimmune hemolytic anemia in a patient with T cell/histiocyte-rich large B cell lymphoma," International Journal of Hematology, vol. 100, no. 5, pp. 490-493, 2014. 
[18] M. B. Jordan, C. E. Allen, S. Weitzman, A. H. Filipovich, and K. L. McClain, "How I treat hemophagocytic lymphohistiocytosis," Blood, vol. 118, no. 15, pp. 4041-4052, 2011.

[19] J. E. Churpek, K. Pyrtel, K.-L. Kanchi et al., "Genomic analysis of germ line and somatic variants in familial myelodysplasia/acute myeloid leukemia," Blood, vol. 126, no. 22, pp. 2484-2490, 2015.

[20] C. C. Coombs, M. S. Tallman, and R. L. Levine, "Molecular therapy for acute myeloid leukaemia," Nature Reviews Clinical Oncology, vol. 13, no. 5, pp. 305-318, 2016.

[21] C. Y. Ok, K. P. Patel, G. Garcia-Manero et al., “TP53 mutation characteristics in therapy-related myelodysplastic syndromes and acute myeloid leukemia is similar to de novo diseases," Journal of Hematology and Oncology, vol. 8, no. 1, article 139, 2015.

[22] M. Tokumasu, C. Murata, A. Shimada et al., "Adverse prognostic impact of KIT mutations in childhood CBF-AML: the results of the Japanese Pediatric Leukemia/Lymphoma Study Group AML-05 trial," Leukemia, vol. 29, no. 12, pp. 2438-2441, 2015. 


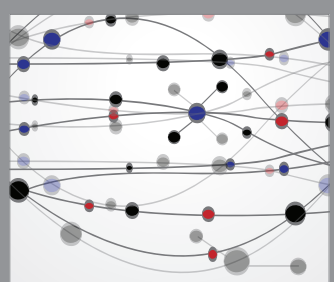

The Scientific World Journal
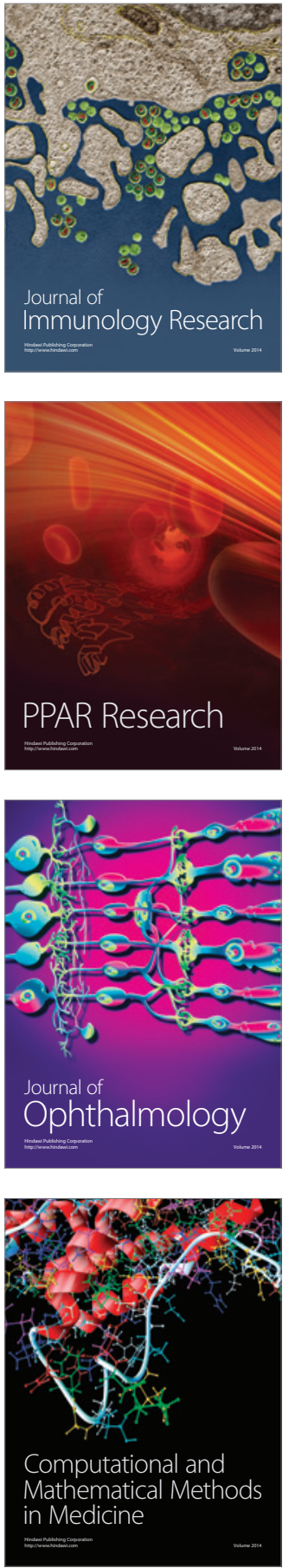

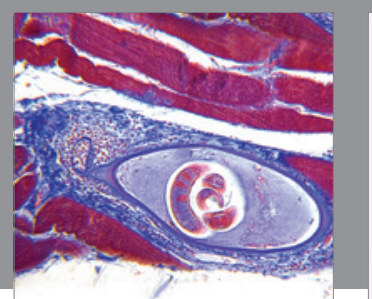

Gastroenterology Research and Practice

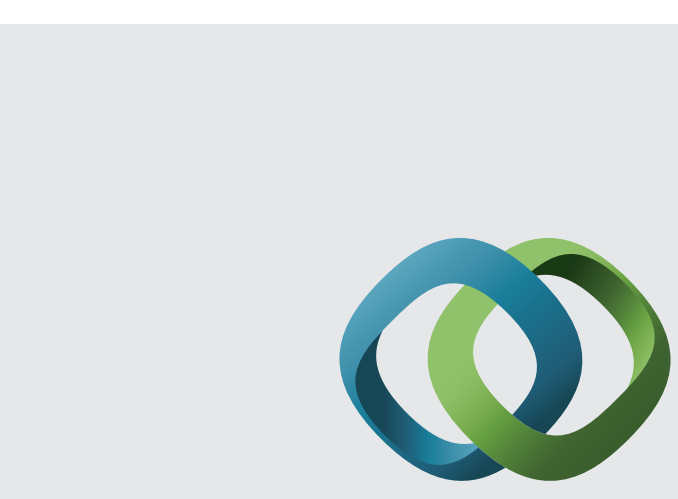

\section{Hindawi}

Submit your manuscripts at

http://www.hindawi.com
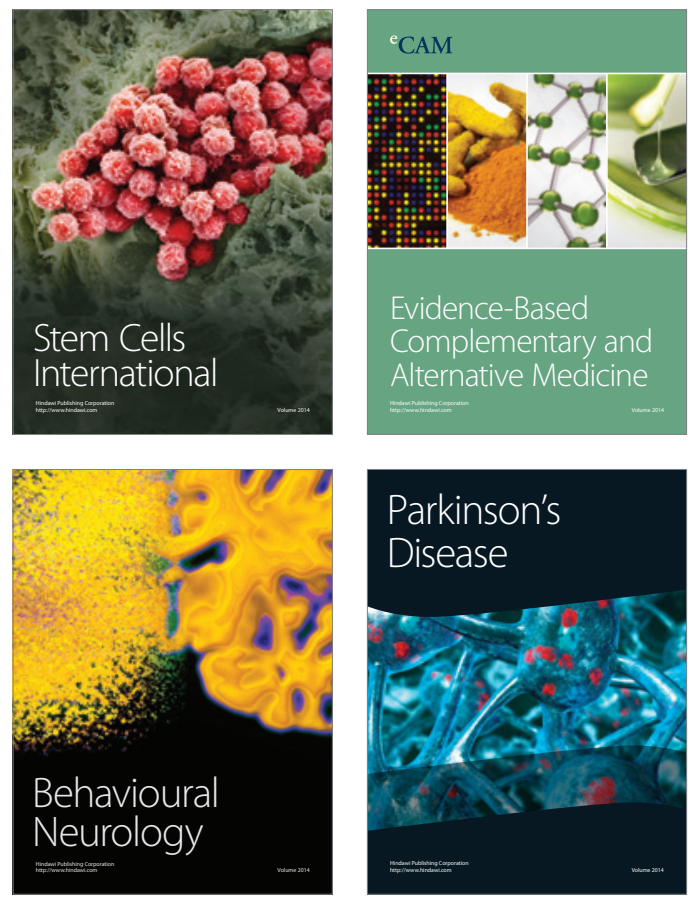
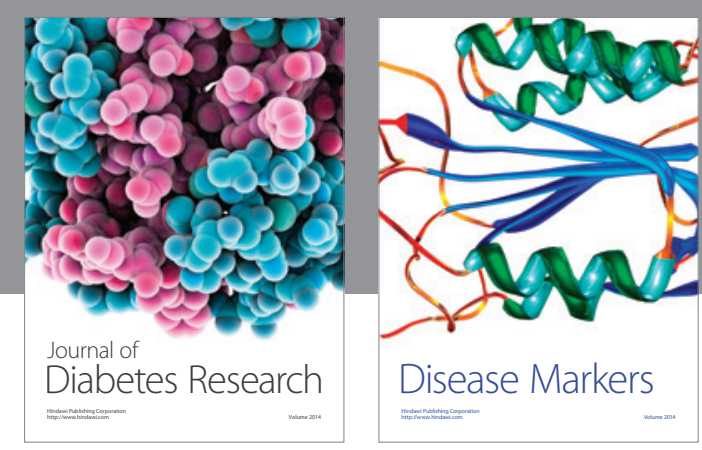

Disease Markers
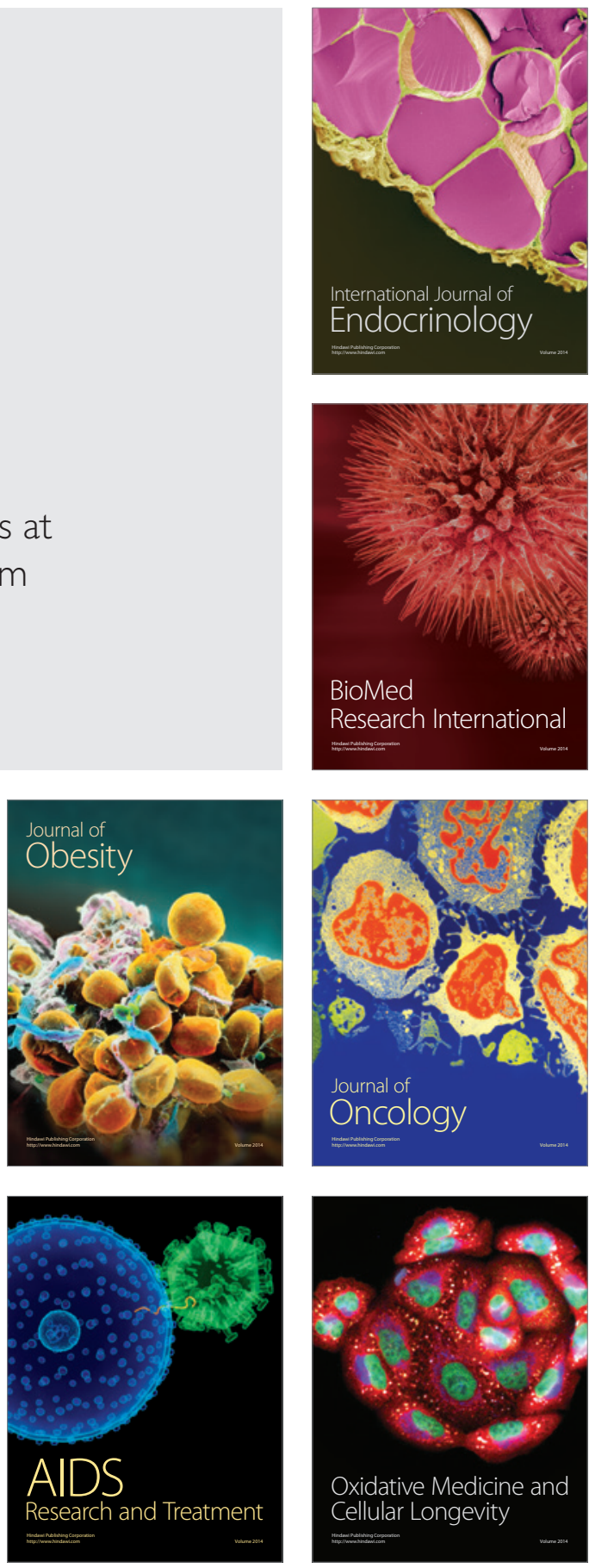